\title{
25. Chemico-physical Research on Water-With Special Reference to Normal, Degenerated and Regenerated Rabbit's Sciatic Nerve (I)
}

\author{
Yoshimasa Isayama and Akio YamanaKa \\ Department of Ophthalmology, School of Medicine, Kobe University \\ Yoshiki INouE and Yukio KanAJI \\ Department of Industrial Chemistry, Faculty of \\ Engeneering, Kobe University
}

About $70 \%$ of the constituents in nervous tissue consists of water, which is assumed to play an important role in its function, whereas little attention to the chemico-physical aspect of their function has been paid previously.

Physico-chemical researches have proved that the molecular characteristic of water has a large dielectric constant and dipole moment, and therefore it has close relation to the intermolecular force by formation of the hydrogenbond and co-ordination of water. It is easily assumed that these properties of water play an important role in the maintainance of the normal structure and function of the nervous tissues. The present experiment will describe about the state of water on the normal, degenerated and regenerated sciatic nerves of rabbits, using the nuclear magnetic resonance (N.M.R.) and absorption of the infra-red spectrum (I.R.S.).

\section{Methods}

A normal rabbit's sciatic nerver were used. Degenerated nerves were taken at $24,48,120$ hours after severance of the nerve. The regenerated one was examined 2.5 months after suturing the cut nerve. The extended nerves were inserted into a sampling tube containing liquid paraffin. The direction of nerve fibers were kept parallel or perpendicular to the tube. N.M.R. of proton of water was measured under $60 \mathrm{Mc}$ at $26^{\circ} \mathrm{C}$, using N.M.R. apparatus type J.N.M. 3H-60. Spinning was not employed in this experiment for the following reasons, namely, (1) the resonance signal of the materials was observed considerably broad, (2) anisotropies were found which were observed by changing the direction of the magnetic field to the never fiber.

The sample of I.R.S. was provided by extending the nerve on K.R.S. -5 cell as thinly as possible.

\section{Results and Discussion}

Figures (A, B, C) are results by N.M.R. obtained from the normal, 
Fig. A

Fig. B

Fig. C
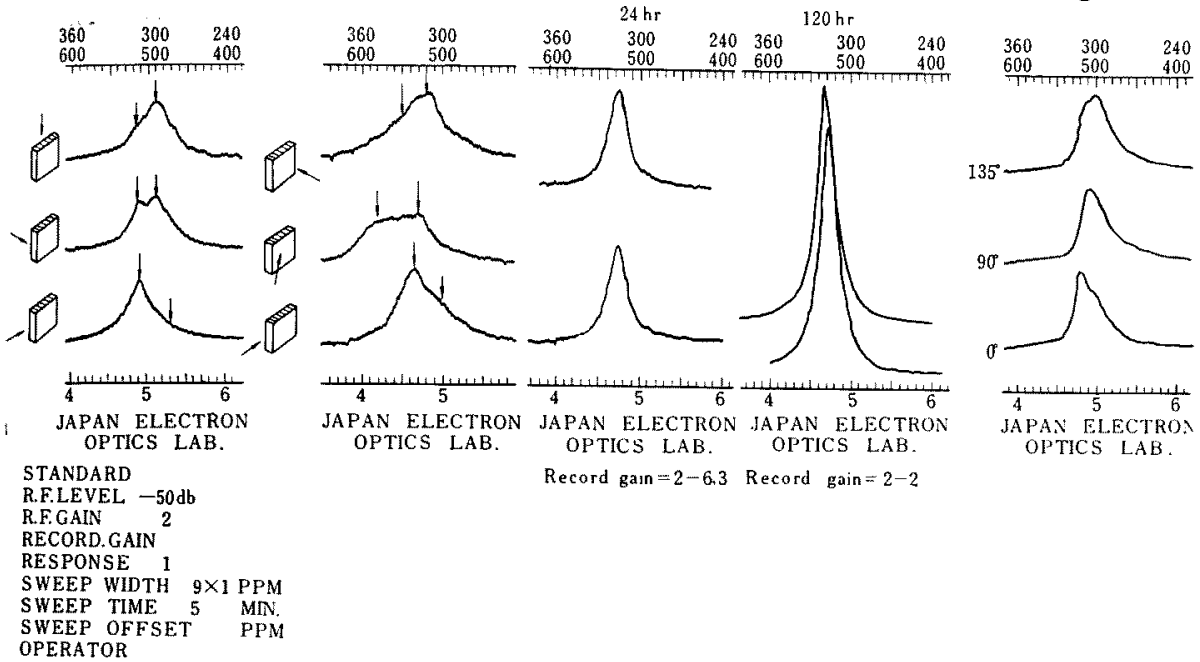

degenerated and regenerated sciatic nerves.

Remarkable change of the resonance position, width and amounts of the signal were observed in accordance with the condition of the nerve respectively. Generally speaking, in the normal nerve, when the direction of the magnetic field $\left(\mathrm{H}_{0}\right)$ was given parallel to the direction of the nerve fiber, the signal of higher field $(\tau \doteqdot 5.10)$ to the free water was observed (Fig. A). When the magnetic field was given perpendicular to the nerve fiber, broad signals of the lower field $(\tau \fallingdotseq 4.9,4.7,4.2)$ were found. Those facts indicate existence of the anisotropy of the resonance signal of water in the normal nerve. But the singlet was obtained in the degenerated nerve, and the anisotropy could not be observed (Fig. B). Splitting of the resonance and the anisotropy appeared gradually, in the course of the regeneration of the nerve (Fig. C). The signal curve of the nerve almost corresponds to the Lorentzian, but the width of each signal differs respectively in the normal, degenerated and regenerated nerves. The half width of the signal in the normal nerve was broad, and this would suggest that bound water exists strongly in the tissue. The half width of the degenerated nerve became narrow and when the nerve regenerates, it becomes wider again. The integrated area of the signal proportionates to the amount of nuclear spin. The amount of signal in the case of the degenerated nerve shows a slight change at 24 hours, but indicates remarkable increase on the 2 nd and 5 th day in the course of degeneration. The above change of signal would indicate that the structure of water which has a characteristic anisotropy in normal nerve break down, and the amount of free water increases at the same time. It seems that the anisotropy above mentioned resulted in the orientation 
and configuration of water. Increase of water in the advanced stage of degeneration would be attributed to the flow of water into the nervous tissue from others. In the region of $\mathrm{OH}$ stretching vibration a shift to low wave number, which is caused by the hydrogen-bond, was observed clearly in I.R.S. of the normal nerve. On the contrary, the absorption spectra were found at the position of free water and the hydrogen-bond shift disappeared in the degenerated nerve.

Those N.M.R. signal patterns typical to the normal, degenerated and regenerated nerver would be indicators of the physico-chemical character of water in the nerve which has close relation to its function.

\title{
26. Nerve Regeneration and Transplantation
}

\author{
Hiroaki Kawakita, Tsuyoshi Fujimoto, \\ Shoji Bando and Daiyu TOYOHARA \\ Dept. of Surgery, Kobe University Medical School
}

\section{A Comparative Study on Reinnervated Neuromusclar Junctions of Motor Nerve from Mouse Gastrocnemius by Electron and Light Microscope}

\author{
Seok Jin KIM \\ Department of Orthopaedic-Surgery, Kyoto University \\ School of Medicine
}

The mouse gastrocnemius was denervated by the way that the same sided sciatic-nerve was burned out with dry-ice at mid-thigh and reserved the continuity of the sciatic-nerve. The gastrocnemius postoperatively was divided by electron microscope and the other, silver stained, was observed by light microscope and the following results were obtained.

1. The first reinnervated motor endplate was found about one month after operation by electron and light microscope and the reinnervating process was almost the reverse course of the denervating process. The Schwann cell which had directly overlain subsynaptic sarcolemma retracted from it and instead of Schwann cell terminal axon invaded the subsynaptic groove 